\title{
EVERY TOPOLOGICALLY AMENABLE LOCALLY COMPACT QUANTUM GROUP IS AMENABLE
}

\author{
AMIN ZOBEIDI
}

(Received 23 February 2012)

\begin{abstract}
We prove that every topologically amenable locally compact quantum group is amenable. This answers an open problem by Bédos and Tuset ['Amenability and co-amenability for locally compact quantum groups', Internat. J. Math. 14 (2003), 865-884].
\end{abstract}

2010 Mathematics subject classification: primary 46L89; secondary 43A07.

Keywords and phrases: locally compact quantum group, amenability.

Amenability of a locally compact group $G$ is equivalent to the existence of invariant means on various subspaces of $L_{\infty}(G)$; in particular, $G$ is amenable if and only if it is topologically amenable, that is, there is a left-invariant mean on $C(G)$, the space of bounded continuous functions on $G$. In [1], Bédos and Tuset considered topological amenability of a locally compact quantum group $\mathbb{G}$, but left the question of whether that is equivalent to amenability of the quantum group $\mathbb{G}$ open. In [4, Theorem 3.6], Runde partially answered the question, by proving the equivalence of amenability and topological amenability for co-amenable locally compact quantum groups. He, in fact, used the existence of a bounded approximate identity in $L_{1}(\mathbb{G})$, to extend a topologically invariant mean to an invariant mean on $L_{\infty}(\mathbb{G})$. Moreover, he observed [4, Remark 3.7] that a weaker condition than co-amenability also gives the results, but again left open the question of the equivalence between the amenability and the topological amenability in the general case.

The main result of this paper proves the equivalence in the general locally compact quantum group setting.

THeorem 1. Let $\mathbb{G}$ be a locally compact quantum group. Then the following are equivalent:

(i) G is amenable;

(ii) $\mathbb{G}$ is topologically amenable.

(c) 2012 Australian Mathematical Publishing Association Inc. 0004-9727/2012 \$16.00 
Let us first introduce the notation and terminology that we will be using in the following. For more details on locally compact quantum groups we refer the reader to $[1,3]$. We denote by $\mathbb{G}=\left(L_{\infty}(\mathbb{G}), \Gamma, \varphi, \psi\right)$ a (von Neumann algebraic) locally compact quantum group in the sense of Kustermans and Vaes [3]. We denote by $L_{1}(\mathbb{G})$ the predual of $L_{\infty}(\mathbb{G})$. The reduced quantum group $C^{*}$-algebra and its multiplier $C^{*}$ algebra will be denoted by $C_{0}(\mathbb{G})$ and $C(\mathbb{G})$, respectively; then $C_{0}(\mathbb{G})$ is a weak* dense $C^{*}$-subalgebra of $L_{\infty}(\mathbb{G})$. We denote by

$$
\operatorname{RUC}(\mathbb{G})=\overline{\operatorname{span}\left\{(\iota \otimes f) \Gamma(x): x \in L_{\infty}(\mathbb{G}), f \in L_{1}(\mathbb{G})\right\}} \mid \cdot \|
$$

the space of right uniformly continuous linear functionals on $L_{1}(\mathbb{G})$.

By the results of [4], we have in general

$$
C_{0}(\mathbb{G}) \subseteq \mathrm{RUC}(\mathbb{G}) \subseteq C(\mathbb{G}) \subseteq L_{\infty}(\mathbb{G})
$$

A locally compact quantum group $\mathbb{G}$ is called amenable if there exists a left (equivalently, right or two-sided) invariant mean on $L_{\infty}(\mathbb{G})$, that is, a state $\tilde{F} \in L_{\infty}(\mathbb{G})^{*}$ such that

$$
\tilde{F}((f \otimes \iota) \Gamma(x))=\tilde{F}(x) f(1)
$$

for all $f \in L_{1}(\mathbb{G})$ and $x \in L_{\infty}(\mathbb{G})$.

The quantum group $\mathbb{G}$ is said to be topologically amenable if there exists a leftinvariant mean on $C(\mathbb{G})$, that is, a state $F \in C(\mathbb{G})^{*}$ such that

$$
F((f \otimes \iota) \Gamma(x))=F(x) f(1)
$$

for all $f \in L_{1}(\mathbb{G})$ and $x \in C(\mathbb{G})$.

We now give the proof of our main result.

Proof of Theorem 1. One direction is trivial. Assume that $F \in C(\mathbb{G})^{*}$ is a left-invariant mean on $C(\mathbb{G})$. Let $\rho \in L_{1}(\mathbb{G})$ be a normal state. Then by [4, Theorem 2.4],

$$
(\iota \otimes) \Gamma(x) \in C(\mathbb{G})
$$

for all $x \in L_{\infty}(\mathbb{G})$. Therefore, we can define a linear functional $\tilde{F}: L_{\infty}(\mathbb{G}) \rightarrow \mathbb{C}$ by

$$
\tilde{F}(x):=F((\iota \otimes \rho) \Gamma(x)) \quad\left(x \in L_{\infty}(\mathbb{G})\right) .
$$

Then, since $\Gamma,(\iota \otimes \rho)$ and $F$ are all positive maps, it follows that their composition $\tilde{F}$ is also positive. Also,

$$
\tilde{F}(1)=F((\iota \otimes \rho) \Gamma(1))=F((\iota \otimes \rho)(1 \otimes 1))=F(1)=1 .
$$

So, $\tilde{F}$ is a state on $L_{\infty}(\mathbb{G})$. We show that $\tilde{F}$ is also left-invariant. Suppose that 
$f \in L_{1}(\mathbb{G})$. Then we have

$$
\begin{aligned}
\tilde{F}((f \otimes \iota) \Gamma(x)) & =F((\iota \otimes \rho) \Gamma((f \otimes \iota) \Gamma(x))) \\
& =F((\iota \otimes \rho)((f \otimes \iota \otimes \iota)((\iota \otimes \Gamma) \Gamma(x)))) \\
& =F((\iota \otimes \rho)((f \otimes \iota \otimes \iota)((\Gamma \otimes \iota) \Gamma(x)))) \\
& =F((f \otimes \iota)((\iota \otimes \iota \otimes \rho)((\Gamma \otimes \iota) \Gamma(x)))) \\
& =F((f \otimes \iota)(\Gamma((\iota \otimes \rho) \Gamma(x)))) \\
& =f(1) F((\iota \otimes \rho) \Gamma(x)) \\
& =f(1) \tilde{F}(x) .
\end{aligned}
$$

Hence, $\tilde{F}$ defines a left-invariant mean on $L_{\infty}(\mathbb{G})$, and so $\mathbb{G}$ is amenable.

The above definitions of amenability seem asymmetric, only based on left invariance. Desmedt et al. proved in [2] that the existence of a left-invariant mean is equivalent to the existence of a right-invariant mean, both of which are equivalent to the existence of a two-sided invariant mean on $L_{\infty}(\mathbb{G})$. But their proof uses the fact that the weak and the norm closure of a convex set in a Banach space are the same, and therefore cannot be applied directly to the case of invariant means on $C(\mathbb{G})$, which is not a dual space in general. So, the equivalence of the existence of left, right and twosided invariant means on $C(\mathbb{G})$ was not known in the general case. But as a corollary to our Theorem 1, and its proof, we obtain the following result.

COROLlary 2. Let $\mathbb{G}$ be a locally compact quantum group. Then the following are equivalent:

(1) $\mathbb{G}$ is amenable;

(2) $C(\mathbb{G})$ has a left-invariant mean;

(3) $C(\mathbb{G})$ has a right-invariant mean;

(4) $C(\mathbb{G})$ has a two-sided invariant mean;

(5) LUC(G) has a right-invariant mean;

(6) RUC(G) has a left-invariant mean.

\section{References}

[1] E. Bédos and L. Tuset, 'Amenability and co-amenability for locally compact quantum groups', Internat. J. Math. 14 (2003), 865-884.

[2] P. Desmedt, J. Quaegebeur and S. Vaes, 'Amenability and the bicrossed product construction', Illinois J. Math. 46 (2002), 1259-1277.

[3] J. Kustermans and S. Vaes, 'Locally compact quantum groups', Ann. Sci. Éc. Norm. Supér. 33 (2000), 837-934.

[4] V. Runde, 'Uniform continuity over locally compact quantum groups', J. Lond. Math. Soc. 80 (2009), 55-71.

AMIN ZOBEIDI, Department of Mathematics, Chamran University, Ahvaz, Iran e-mail: zobeidiamin@gmail.com 